\title{
Alteraciones del tamaño y de la configuración craneana en el lactante
}

\author{
M.C. Germán Schlager C.'
}

\section{Abnormal cranial size or form in infants}

\begin{abstract}
Abnormalities of craneal size and configuration include macrocephaly, microcephaly and dysmorphic skull. Hydrocephalus, megaencephalus, abnormal growth of skull bones and chronic subdural and epidural collections are among the most important causes of macrocephaly. Primary microcephaly may be due to deficient neuronal projiferation or abnomalities in neural tube segmentation or celular migration. Secondary microcephaly may be caused by prenatal, perinatal or early postnatal insults like infections, drugs, radiation, hipoxia, among others. Abnormal cranial forms may be sporadic or secondary to genetic and chromosomal diseases. Craneosynostosís can be managed surgically with positive results when early diagnosis and treatment are accomplished.

(Key words: Macrocephaly, microcephaly, dysmorphic skull.)
\end{abstract}

El crecimiento del cráneo está claramente relacionado con el crecimiento del encéfalo durante la niñez y es importante y rápido durante el período perinatal y en los dos primeros años de vida. El perímetro cefálico, medido sobre la arcada ciliar por delante, pasando por la prominencia occipital por atrás, es un buen indice del tamaño cerebral. En el recién nacido de término el perímetro craneano es de $35 \pm 1,5 \mathrm{~cm}$ $y$ al año de edad alcanza a $47 \pm 2,2 \mathrm{~cm}$, siendo algo más pequeño en las niñas que en los nifros. En el recién nacido pretérmino el perímetro craneano inicial y su crecimiento guardan relación con la edad gestacional ${ }^{1}$.

El recién nacido o el lactante con una cabeza anormalmente grande o pequefta constituye a menudo un problema de defícil diagnóstico. Es muy importante determinar con mediciones periódicas del perimetro craneano si existe un crecimiento anormal, por encima o por debajo de lo esperado, en lo posible cotejándolo y registrándolo en una curva de crecimjento normal. Durante los tres primeros meses de vida, en el recién nacido de término se encuentra un crecimiento de $2 \mathrm{~cm}$ por mes, que disminuye durante el segundo trimestre a $1 \mathrm{~cm}$ por mes $y$ el tercer y cuarto trimestre a $0,5 \mathrm{~cm}$ por mes. El crecimiento del perimetro craneano en el prematuro muestra algunas diferencias con el que podría esperarse en el recién nacido de término y con el crecimiento intrauterino: des-

1. Clínica Alemana, Servicio de Pediatría. pués de 2 a 4 semanas de crecimiento moderado $(0,7 \mathrm{~cm}$ por semana), que lleva a medidas algo inferiores a las correspondientes para igual edad concepcional en la curva de crecimiento intrauterino, se observa un período de crecimiento acelerado: aumenta en promedio 4,2 $\mathrm{cm}$ entre las 33 y 36 semanas de edad concepcional y $2,4 \mathrm{~cm}$ entre las 37 y 40 semanas, lo que lleva a medidas algo superiores a las correspondientes a la curva de crecimiento intrauterino ${ }^{2}$.

La comprobación de cráneo pequeño en un lactante o recién nacido es muy sugerente de insuficiente crecimiento cerebral, aislado o formando parte de un déficit global de crecimiento. Debe excluirse la posibilidad de eierre precoz de suturas (craneosinostosis), trastorno oseo que puede llevar secundariamente a atrofia cerebral por limitación de su expansión y por compresión por aumento crónico de la presión intracraneana. Un crábeo grande, más de dos desviaciones estándar sobre la media para la edad y el sexo correspondientes, tiene habitualmente como causa la acumulación anormal de líquido cefalo. rraquídeo (LCR) y sólo en raras ocasiones se debe a crecimiento cerebral exagerado (megaencefalia) o a alteraciones óseas.

La forma del cráneo está en parte determinada por su contenido, pero también fuerzas externas que actúan sobre él especialmente a fines del período de gestación $y$ en el parto, asi como la fusión anormal de los huesos craneanos tienen un papel de importancia ${ }^{3}$. 


\section{MACROCEFALIA}

Las principales causas de macrocefalia son hidrocefalia (excesivo volumen de LCR en la cavidad craneana), megaencefalia (aumento de tamaño del cerebro), engrosamiento del cráneo y hematomas crónicos en los espacios subdural o epidural.

Las hidrocefalias usualmente se clasifican en comunicantes (no hay obstrucción a la circulación del LCR) y no comunicantes (hay una obstrucción en el sistema ventricular que impide la circulación del LCR). Las megaencefalias también se dividen en dos categorías: megaencefalia anatórnica (megaencefalia primaria y sindromes neurocutáneos) y megaencefalia metabólica (secundarja a edema crónico y acumulación de productos metabólicos). El engrosamiento o la alteración del crecimiento de los huesos craneanos puede deberse a enfermedades generales o a trastornos propios del desarrollo óseo. Los hematomas crónicos tienen habitualmente su origen en traumatismos encefalocraneanos, excesivo moldeado o traumatismos durante el parto $\mathrm{y}$ deshidratación o in. fecciones postnatales.

Hidrocefalia comunicante. Habitualmente es causada por disminución de la reabsorción de LCR en la convexidad craneana, debida a inflamación meníngea crónica, distorsión, bloqueos y fibrosis del espacio subaracnoideo o infiltración tumoral. El exceso de producción de LCR por un papiloma del plexo coroideo es muy poco frecuente. Hay también algunos trastomos genéticos o constitucionales que considerar, entre ellos la acondroplasia y el agrandamiento benigno del espacio subaracnoideo.

La acondroplasia es un trastorno genético, autosómico dominante, que condiciona enanismo por acortamiento proximal de las extremidades y se asocia a macrocefalia. Debido a que los senos venosos de la base del cráneo son de tamaño muy reducido en estos pacientes, hay disminución del retomo venoso, aumento de la presión venosa y menor reabsorción del LCR, que causará hidrocefalia comunicante con tendencia espontánea a estabilizarse, que taras veces requiere de procedimientos quirúrgicos de derivación ${ }^{3}$.

E1 agrandamiento benigno del espacio subaracnoideo, conocido también como hidrocéfalo externo, es relativamante común. Muchas veces hay una historia familiar de mactocráneo, con perímetros craneanos cercanos al percentil 97, canal de crecimiento en el que se mantienen. La tomografía axial computarizada (TAC) muestra ensanchamiento de los surcos y cisuras cerebrales y ensanchamiento del espacio subaracnoideo, especialmente en la región frontal (donde se acepta hasta $5,7 \mathrm{~mm}$ como el liquido normal), conservándose el tamafio ventricular sin alteraciones. Su pronóstico espontáneo es bueno y no requiere tratamiento, salvo si el cre. cimiento craneano continúa y llega a exceder el percentil 97, caso en que se justifica repetir la tomografía computadorizada y, eventualmente, decidir la colocación de una derivación hacia el peritoneo 4,5 .

Hidrocefalia no comunicante. En estos casos hay interferencia con la circulación del LCR en el sistema ventricular mismo, con acumulación retrógrada de aquél y dilatación secundaria de los ventrículos. El agrandamiento ventricular puede preceder por período variables de tiempo al crecimiento craneano excesivo y rápido, que es la manifestación clínica más conocida de hidrocefalia. La macrocefalia puede ser evidente en el útero, en el período neonatal o en los primeros meses de vida. El niño tiene la frente grande y sobresaliente, la piel del cráneo estirada, aumento de la circulación venosa, fontanela anterior amplia y tensa y suturas separadas. Los ojos tienden a desviarse hacia abajo, por paresia de la supraversión ocular debida a compresión del tectum mesencefálico (signo del sol poniente). Los reflejos elementales y los patrones conductuales básicos del recién nacido y del lactante aparecen preservados, pero en las hidrocefalias avanzadas hay signos de compromiso corticoespinal y corticobulbar. Ocasionalmente en el recién nacido se encuentra un déficit casi total del desarrollo de los hemisferios cerebrales, o su desaparición casi completa, conservándose las estructuras de la fosa posterior, condición conocida con el nombre de hidranencefalia ${ }^{6}$.

El tratamiento de las distintas formas de hidrocefalia consiste en proporcionar una ruta alternativa al LCR, desđe uno de los ventrículos cerebrales a la vena cava superior o a la cavidad peritoneal. El uso de un inhibidor de la anhidrasa carbónica, la acetazolamida, disminuye en $50 \%$ la producción de LCR en los plexos coroideos, proporcionando alivio transitorio en los casos en que deba posponerse la intervención quirúrgica. 
Las malformaciones que con más frecuencia se encuentran como causa de hidrocefalia son la estenosis congénita del acueducto, la malformación de Arnold-Chiari y la malformación de Dandy-Walker. La primera es causada por un defecto de la orgarogénesis y a veces por obstrucción inflamatoria del acueducto, que lleva a una hidrocefalia triventricular, con dilatación de los ventrículos laterales y del tercer ventrículo de rápida evolución. La malformación de Amold-Chiari se encuentra en la mayoría de los pacientes con meningomielocele y consiste en el desplazamiento caudal del bulbo raquídeo y del IV ventrículo, alargamiento del tronco cerebral $\mathrm{y}$ desplazamiento del cerebelo a través del foramen magno. Esta asociación de malformaciones de la fosa posterior, al obstruir la circulación del LCR, causa dilatación de los ventrículos laterales y del III y IV ventrículo. La malformación de Dandy-Walker consiste en una dilatación quística del IV ventrículo, acompañada habitualmente de atresia de los agujeros de Luschka y Magendie, y en 25\% de los casos de agenesia del vermis cerebeloso. Es causada por un defecto en la formación del techo del rombencéfalo $y$ puede acompañarse de otras malformaciones del encéfalo ${ }^{1,2}$.

Megaencefalia. La megaencefalia anatómica incluye aquellas condjciones en las cuales el cerebro está agrandado como resultado de aumentos de número o tamaño de las células, sin evidencias de encefalopatía o de enfermedad metabólica. La megaencefalia genética abarca aquellas familias en que se presentan macrocefalias, con pe. rímetros craneanos alrededor del percentil 97, sin otras anormalidades somáticas y con examen neurológico $\mathrm{y}$ función intelectual nomales. Pueden diferenciarse de aquellos nifíos con agrandamiento benigno del espacio subaracnoideo mediante tomografía computadorizada. La megaencefalia con gigantismo es también deno. minada gigantismo cerebral o síndrome de Sotos. Algunos casos son esporádicos y otros genéticos, transmitidos por herencia autosómica recesiva o dominante. Los estudios cromosómjcos son normales. Se trata de niños macrosómicos y de rápido crecimiento hasta los 3 aftos de edad, con frente prominente, hipertelorismo y paladar ojival. El $80 \%$ tiene algún grado de re. tardo mental. La tomografía computadorizada só lo muestra un discreto aumento del tamanto ventricular y los estudios endocronológicos son normales. Algunos pacientes con enfermedades neuro. cutáneas, como la neurofibromatosis y la esclerosis tuberosa, tienen macrocefalia, que se hace evidente durante su desartollo, y que es causada en algunos casos por hidrocefalia y en otros por megaencefalia. Puede encontrarse hemimegaencefalia, hemihipertrofia corporal o hipertrofia de una sola extremidad. Obviamente, el hallazgo de estas anormalidades del crecimiento en un nifio aparentemente sano debe alertar sobre la posibilidad de que padezca de una enfermedad neurocutánea ${ }^{7}$.

La megaencefalia metabólica es causada por el almacenamiento intracelular de sustancias anormales o por edema cerebral persistente, en el curso de algunas enfermedades debidas a errores congénitos del metabolismo. Cabe destacar las enfermedades de Canavan y de Alexander, la galactosemia, las gangliosidosis, las leucodistrofias, las mucopolisacaridosis y las enfermedades de orina con olor a jarabe de arce. El agrandamiento cerebral se acompaña paralela. mente de regresión psicomotora y de hiperten. sión intracraneana. En la tomografía computadorizada se evidencia agrandamiento cerebral con ventriculos pequefios ${ }^{6}$.

Trastomos del desarrollo de los huesos craneanos. Cabe mencionar la osteopetrosis, la displasia metafisiaria, la condrodistrofia calcificante y especialmente la disostosis cleidocraneal. Esta última puede confundirse făcilmente con hidrocefalia, ya que suele manifestarse con importante macrocefalia y fontanela antėrior muy amplia ${ }^{3}$.

Colecciones yuxtadurales crónicas. Las colecciones yuxtadurales son causa frecuente de aumento del tamafio craneano y de hipertensión intracraneana en el lactante. La más frecuente y de mayor importancia clínica es el hematoma subdural crónico, aunque también puede producirse en acumulaciones subdurales de LCR (hidroma), de líquido hiperproteico en el transcurso de meningitis (higroma) o de pus (empiema subdural). Los hematomas subdurales crónicos tienen una evolución de más de diez días a partir de hemorragias de origen traumático o causadas por roturas de venas puente en deshidrataciones hipertónicas. Mediante punción subdural pueden extraerse más de $2 \mathrm{~m}$ ] de liquido, cuya concentración de proteínas es de por lo menos $40 \mathrm{mg} / \mathrm{dl}$ mayor que la del LCR obtenido por punción lumbar, con menos de 1 millón de glóbulos rojos por $\mathrm{mm}^{3}$. En 31\% de los lactantes con hematoma subdural crónico existe 
macrocefalia y 63\% tienen fontanela tensa y separación de suturas. El tratamiento actualmente es conservador, ya que la mayor parte de las veces las colecciones subdurales se reab. sorben espontâneamente. Las colecciones mayores y muy crónicas pueden requerir de punciones subdurales repetidas y los empiemas subdurales de vaciamiento quirúrgico ${ }^{8}$.

\section{MICROCEFALIA}

Un perimetro craneano inferior en dos o más desviaciones estándar a la media para la edad y sexo del niño define la presencia de microcefalia. Sus causas son múltiples, pudiendo tratarse de defectos en el desarrollo del cráneo, con ciêrre precoz de fontanela y suturas o de trastornos del crecimiento cerebral, ya sea por defectos de la organogénesis cerebral (microencefalias primarias) o como secuelas de afecciones prenatales, del parto, neonatales o del lactante con dano importante del tejido neural (microencefalias secundarias). En los niños con microcefalia el examen neurológico puede ser normal o mostrar variados signos de disfunción dentro de un amplio rango de severidad. Casi siempre se acompanta de retardo mental, pudiendo decirse que hay una relación directa y lineal entre el grado de retraso intelectual y la severidad de la microcefalia'.

Microcefalias primarias. La llamada microencefalia vera es causada por déficit de proliferación neuronal, habitualmente heredados en forma autosómica dominante o recesiva. Los recjén nacidos afectados presentan cráneos pequeños, con escaso crecimiento ulterior. Esta forma de microencefalia debe diferenciarse de otros defectos de la organogénesis cerebral y de las formas secundarias, siendo particularmente ütì, para este efecto, la TAC, que muestra que el cerebro es pequeño pero de aspecto normal.

Los defectos del proceso de vesiculación en la zona anterior del tubo neural producirán malformación de] prosencéfalo, con agenesia del cuerpo calloso, ventrículo único cubierto por corteza límbica, microencefalia y malformaciones faciales (ciclopia, hipotelorismo, labio leporino). Los casos más severos corresponden a mortinatos o a recién nacidos que fallecen ulteriormente, pero los más leves, en los cuales hay solamente menor desarrollo del rinencéfalo y de los bulbos olfatorios, suelen tener aspecto normal, microencefalia moderada, retraso leve del desartollo psicomotor y a veces epilepsia.

Los trastornos de la migración celular, con migración defectuosa e incompleta de las neuronas destinadas a las capas más superficiales de la corteza cerebral, pueden traducirse en alteracio. nes de la corteza (agiria, paquigiria) y en acumulación anornal de neuronas en la sustancia blanca (heterotopias). La mitad de los casos tiene microcefalia, pero casi todos presentan retraso del desarrollo psicomotor y crisis convulsivas ${ }^{10}$.

Microcefalias secundarias. De las causas pre. natales, las infecciones intrauterinas por virus son frecuentes e importantes, destacando el virus de la rubéola y el citomegalovinus. Algunas drogas, tóxicos y radiaciones también pueden praducir microcefalia, asociada muchas veces a retardo del crecimiento corporal y a caracteristicas dismórficas (como el síndrome alcohólico fetal, el sindrome hidantoinico, la exposición a rayos $X$, etc.).

Las lesiones cerebrales perinatales son la causa más común de crecimiento cerebral deficiente en niños cuyo perímetro craneano al nacer era normal. Deben considerarse especialmente las secuelas de la encefalopatía anóxico-isquémica $y$ de las hemorragias intracraneanas. Jamás puede omitirse la medición del perímetro craneano en los recién nacidos, verificándola varias veces, de ser necesario, en neonatos con cefalohematomas o moldeado craneano excesivo y registrándola apropiadamente, junto con el peso, la talla y la puntuación de Apgar en el camé del recién nacido. Los niños con lesiones cerebrales de origen perinatal, además de microcefalia, presentan parálisis cerebral, retardo mental o crisis convulsivas asocíadas ${ }^{i, 2}$.

Entre las enfermedades postnatales, especial. mente del periodo neonatal o del lactante menor, están las infecciones que pueden producir destrucción del tejido neural (meningitis y encefalitis), las enfermedades crónicas de otros órganos y sistemas y la desnutrición precoz y grave.

\section{ALTERACIONES DE LA FORMA CRANEANA}

La forma del cráneo no sólo depende de su contenido sino también de fuerzas de moldeado externo operantes en el útero o el transcurso del parto y del cierre oportuno y amónico de las diferentes suturas craneanas. Las alteraciones de la forma del cráneo, sean aisladas o formando 
parte de un trastorno del crecimiento mismo, son esporádicas en la mayoría de los casos, pero también pueden corresponder a trastornos genéticos o cromosómicos, como deleciones parciales o sindromes conocidos. Por sus características destacan la disostosis cráneo-facial o enfermedad de Crouzon y la acrocefalosindactilia o síndrome de Apert ${ }^{3}$.

Craneoestemosis. Los términos "craneostenosis" y "craneosinostosis" describen el cierre precoz de una o más suturas craneanas, lo que determinará una forma craneana anormal. El cierre precoz de las fontanelas y suturas en los niños con microcefalia se debe a la falta de la presión intracraneana normal necesaria para mantener las suturas con su separación fisiológica. En las craneosinostosis que afectan varias suturas se producirá microcefalia por el déficit de crecimiento óseo, con constricción del crecimiento cerebral, síntornas de hipertensión intracraneana y daño cerebral secundario. La alteración más comúnmente encontrada de la forma del cráneo es la escafocefalia, causada por la fusión prematura de la sutura sagital, lo que hace crecer al cráneo muy alargado en sentido anteroposterior. La fusión prematura de ambas suturas coronales llevará a una braquicefalia y la fusión de sólo una sutura coronal o lambdoidea llevará a un desarrollo asimétrico conocido como plagiocefalia ${ }^{1-3}$.

En los casos en que hay fusión de varias suturas y el crecimiento craneano es insuficiente, con signos de hipertensión intracraneana y riesgo de atrofia cerebral secundaria, es necesario efectuar su corrección quirúrgica, practicando suturas artificiales mediante incisiones óseas paralelas a las suturas cerradas y permitiendo así la reexpansión cerebral y la continuación del crecimiento craneano. Ocasionalmente esta intervención puede ser necesaria por razones cosméticas, especialmente en plagiocefalias muy severas y deformantes.

\section{RESUMEN}

Las principales alteraciones de] tamaño $y$ de la configuración craneana en el lactante pue- den clasificarse en macrocefalia, microcefalia y alteraciones de la forma del cráneo. Entre las principales causas de macrocefalia están la hidrocefalia, megacefalia, el engrosamiento de las paredes del cráneo y los hematomas crónicos subdurales y epidurales. Entre las microcefalias destacan las primarias por fallas de prolifera. ción neuronal, defectos del proceso de vesiculación del tubo neural y los trastornos de la migración celular. Las microcefalias secundarias más frecuentes son aquellas debidas a infecciones intrauterinas, drogas, tóxicos, radiaciones, hipoxia e infecciones postnatales. Entre las alteraciones de la forma del cráneo son frecuentes las esporádicas y las debidas a trastornos genéticos o cromosómicos, siendo las craneoestenosis una de las más frecuentes y susceptibles de corrección quirúrgica con resultados positivos si el diagnóstico se hace precozmente.

(Palabras clave: microcefalia, macrocefalia, dismorfias craneanas.)

\section{REFERENCIAS}

1. Clifford-Rose F.: Paediatric Neurology. Blackwell Sc. Public. Oxford, 1979.

2. Schlager $G$.: Neuropediatría 1: Neurología đel reciến nacido. Ed. Andrés Bello, Santiago, 1983.

3. Jacobson R.: Abnormalities of the skull in children. Neurologic Clinics 1985; 3: 117-144

4. Lorber J., Priestly B.: Children with large heads: A practical approach to diagnosis in 557 children with special reference to 109 children with megaencephaly. Dev Med Child Neurol 1981; 23: 494.

5. Weaver $D$., Christian $J$.: Familial variation of head size and adjustment for parenteral head circumference. J Pediatr 1980; 96: 990

6. Donat $J_{\text {. }}$ Evaluation of macrocranea using computed tomography. Am J Dis Child 1981;135:1118.

7. De Myer W.: Megalencephaly: types, clinical syndromes and management. Pediatr Neurol $1986 ; 2: 321$.

8. Hamza M., Bodensteiner J., Noorani P.: Benign extracerebral fluid collections: A cause of macrocranea in infancy. Pediatr Neurol 1987; 3: 218.

9. Jaworski M., Hersh J., Donat J.: Computed tomography of the head in the evaluation of $\mathbf{m i}$ crocephaly. Pediatrics 1986; 78 : 1064 ,

10. Jellinger $K$., Gross $H_{\text {, }}$ Kaltenbach $E$.: Holoprosencephaly and agenesis of the corpus callosum. Acta Neuropathol 1981; $55: 1$. 